IJMMS 25:9 (2001) 609-619

PII. S0161171201001028

http://ijmms.hindawi.com

(C) Hindawi Publishing Corp.

\title{
RAYLEIGH-BENARD CONVECTION IN A VISCOELASTIC FLUID-FILLED HIGH-POROSITY MEDIUM WITH NONUNIFORM BASIC TEMPERATURE GRADIENT
}

\author{
PRADEEP G. SIDDHESHWAR and C. V. SRI KRISHNA
}

(Received 16 February 1998)

\begin{abstract}
The qualitative effect of nonuniform temperature gradient on the linear stability analysis of the Rayleigh-Benard convection problem in a Boussinesquian, viscoelastic fluid-filled, high-porosity medium is studied numerically using the single-term Galerkin technique. The eigenvalue is obtained for free-free, free-rigid, and rigid-rigid boundary combinations with isothermal temperature conditions. Thermodynamics and also the present stability analysis dictates the strain retardation time to be less than the stress relaxation time for convection to set in as oscillatory motions in a high-porosity medium. Furthermore, the analysis predicts the critical eigenvalue for the viscoelastic problem to be less than that of the corresponding Newtonian fluid problem.
\end{abstract}

2000 Mathematics Subject Classification. Primary 76-XX.

1. Introduction. The classical Rayleigh-Benard problem in porous media, with no viscoelastic effects, has received considerable attention (see Nield [10], Rudraiah [20], Poulikakos [16] and references therein) and it was shown that instability appears in the direct mode.

In a series of papers [12, 13, 14], Oldroyd proposed and studied a set of constitutive equations for viscoelastic fluids in an attempt to explain the rheological behaviour of some non-Newtonian fluids. The dynamical behaviour of these practically important fluids under isothermal conditions is better understood than under nonisothermal conditions (see Harris [5]). In the Rayleigh-Benard instability problem with respect to these fluids, the principle of exchange of stability is, in general, not valid and hence oscillatory mode of convection appears.

Herbert [6] was the first to analyse the problem of oscillatory convection in Oldroyd viscoelastic fluids. Subsequently, Green [4], Vest and Arpaci [22] studied the problem using the not-very-realistic Jeffrey and Maxwell models. Sokolov and Tanner [21] confirm the unrealistic nature of these models by using an integral form of constitutive equation. Eltayeb [3] made an elaborate linear and nonlinear analysis of the problem using the Oldroyd model and brought out the deficiencies in the works based on Jeffrey and Maxwell models. Recently, Rosenblat [17], Mardones and Garcia [9] have also made a study of the problem. More recently, Park and Lee [15] have investigated the Hopf bifurcation of viscoelastic fluids heated from below with rigidrigid and rigid-free boundary conditions for a range of viscoelastic parameters where 
Hopf bifurcations occur. The nonlinear analysis based on power-series method reveals that various parameters have significant effects on hydrodynamic stability and suggests that the Rayleigh-Benard convective system may be used at least, in part, as a useful rheometric tool to assess the suitability of constitutive equations. All the aforementioned works concern purely viscous fluid layers (i.e., no porous medium).

The oscillatory mode of convection in viscoelastic fluids occupying high-porosity media is of practical interest. The problem is characterised by large values of the effective Prandtl number and hence the line of thinking of Poulikakos [16], used in the context of Newtonian fluids, may be appropriately extended to non-Newtonian fluids. As a result the local and convective accelerations have to be dropped from the momentum conservation equation. The earlier works on convection in viscoelastic fluids occupying porous media (see $[18,19]$ ) concern densely packed beds or sparsely packed beds with local and convective accelerations. Rudraiah et al. [19] also assume the fluid viscosity $\mu$ and the effective fluid viscosity $\mu^{\prime}$ to be the same. Poulikakos [16], Lauriat and Prasad [7], and others consider the two to be different in the case of Newtonian fluid flows through porous media and seems the right thing to do in non-Newtonian fluids as well. This tentative observation is made considering the fact that a firm statement in terms of an explicit equation for ratio of viscosities as a function of porosity or permeability is, as yet, unavailable. The values for the ratio of viscosities have thus been a matter of personal opinion and general observation. A detailed discussion on the values of $\mu^{\prime}$ has been presented by Lundgren [8].

The purpose of the present work is to investigate the viscoelastic effects on the onset of Rayleigh-Benard convection in a three-dimensional, horizontal layer of an Oldroydian, Boussinesquian viscoelastic fluid layer occupying a high-porosity medium.

The boundary combinations considered are free-free, free-rigid, and rigid-rigid boundaries which are maintained at constant temperature (isothermal boundary condition). Illustrative numerically calculated values of the critical eigenvalue for a few choices of the parameter values are presented in the form of graphs. The problem has many possible applications in situations where fluids are used as a working medium and porous media as dampers.

2. Mathematical formulation. Consider a horizontal porous layer of infinite extent occupied by a Boussinesquian, viscoelastic fluid of depth $d$. Let $\Delta T$ be the temperature difference between lower and upper flat fluid surfaces. The fluid is assumed viscoelastic and describable by the Oldroyd constitutive equation. The porous medium is assumed to have high-porosity and hence the fluid flow is governed by the Brinkman model with viscoelastic correction. An appropriate single-phase heat transport equation is chosen with effective heat capacity ratio and effective thermal diffusivity. Thus the governing equations for the Rayleigh-Benard situation in a Boussinesquian viscoelastic fluid-saturated porous medium are

$$
\begin{gathered}
q_{i, i}=0, \\
0=-p_{, i}-\rho g \delta_{i 3}-\frac{\mu_{f}}{K} q_{i}+\tau_{i j, j}^{\prime},
\end{gathered}
$$




$$
\begin{gathered}
\left(1+\lambda_{1} \frac{D}{D t}\right) \tau_{i j}^{\prime}=\left(1+\lambda_{2} \frac{D}{D t}\right) \mu^{\prime}\left[\left(q_{i, j}+q_{j, i}\right)\right], \\
M \frac{\partial T}{\partial t}+q_{j} T_{, j}=\chi_{e} T_{, j j} \\
\rho=\rho_{0}\left[1-\alpha\left(T-T_{0}\right)\right],
\end{gathered}
$$

where $q_{i}$ is the filter velocity vector component, $M$ is the heat capacity ratio given by

$$
M=\frac{(1-\Phi)\left(\rho C_{p}\right)_{\text {solid }}+\Phi\left(\rho C_{p}\right)_{\text {fluid }}}{\left(\rho C_{p}\right)_{\text {fluid }}}
$$

and the effective thermal diffusivity $\chi_{e}$ is given by

$$
\chi_{e}=\Phi \chi_{\text {fluid }}+(1-\Phi) \chi_{\text {solid }}
$$

$\Phi$ is the porosity and $\mu^{\prime}$ is the effective viscosity of the porous medium. In (2.2), $K$ is the permeability of the porous medium and is related to $\Phi$ by the Karman-Cozeny relation

$$
K=\frac{d_{p}^{2} \Phi^{2}}{150(1-\Phi)^{2}}
$$

In (2.6), $C_{p}$ is the specific heat at constant pressure.

Eliminating $\tau_{i j}^{\prime}$ between (2.2) and (2.3) and using (2.1), we get

$$
\left(1+\lambda_{1} \frac{D}{D t}\right)\left[p_{, i}+\rho g \delta_{i 3}+\frac{\mu_{f}}{K} q_{i}\right]=\mu^{\prime}\left(1+\lambda_{2} \frac{D}{D t}\right) q_{i, j j}
$$

The local and convective accelerations have been dropped on reasons given by Poulikakos [16].

BASIC STATE. The basic state of the fluid is quiescent and is described by

$$
q_{i b}=(0,0,0), \quad-\frac{d}{\triangle T} \frac{d T_{b}}{d z}=f(z), \quad \rho=\rho_{b}(z), \quad p=p_{b}(z),
$$

where $f(z)$ is the basic nonuniform temperature gradient. The various nonuniform basic temperature gradients considered in this paper are presented in Figure 2.1.

3. Linear stability analysis. The basic state is disturbed by an infinitesimal thermal perturbation, thereby leading to the following equations for the perturbation:

$$
\begin{gathered}
\left(1+\lambda_{1} \frac{\partial}{\partial t}\right)\left[\frac{1}{\mu_{f}} p_{, i}^{\prime}-\frac{\alpha g T^{\prime} \delta_{i 3}}{v}+\frac{1}{K} q_{i}^{\prime}\right]=\Lambda\left(1+\lambda_{2} \frac{\partial}{\partial t}\right) q_{i, j j}^{\prime}, \\
M \frac{\partial T^{\prime}}{\partial t}-W^{\prime} \frac{\Delta T}{d} f(z)=\chi_{e} T_{, j j}^{\prime} .
\end{gathered}
$$

To obtain (3.1) we used the basic state equation and expression for $\rho^{\prime} / \rho_{0}$ from (2.5). 


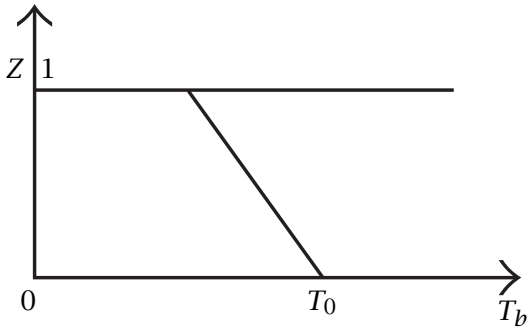

(a) Model-1: linear $[f(z)=1]$.

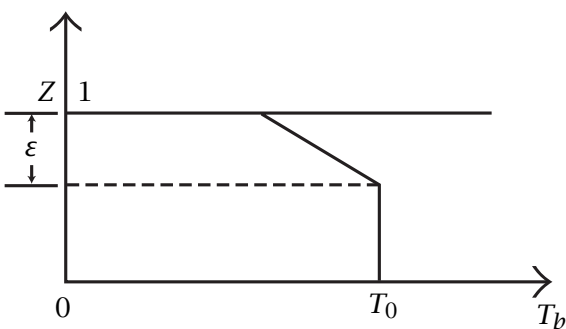

(c) Model-3: piecewise linear (cooled from above) $[f(z)=0$ for all $0 \leq Z$ $<1-\varepsilon$ and $f(z)=\varepsilon^{-1}$ for all $1-\varepsilon<$ $Z \leq 1]$.

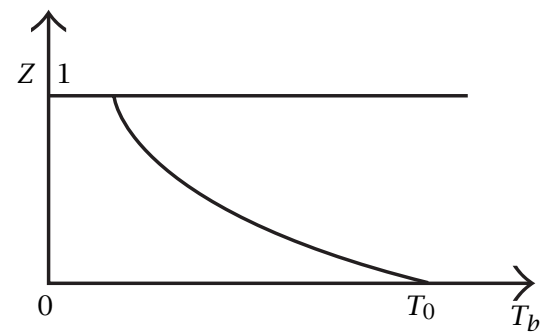

(e) Model-5: inverted parabola, $[f(z)=2(1-Z)]$.

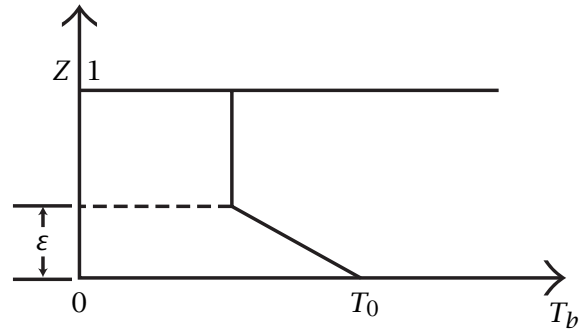

(b) Model-2: piecewise linear (heated from below) $\left[f(z)=\varepsilon^{-1}\right.$ for all $0 \leq Z$ $<\varepsilon$ and $f(z)=0$ for all $\varepsilon<Z \leq 1$ ].

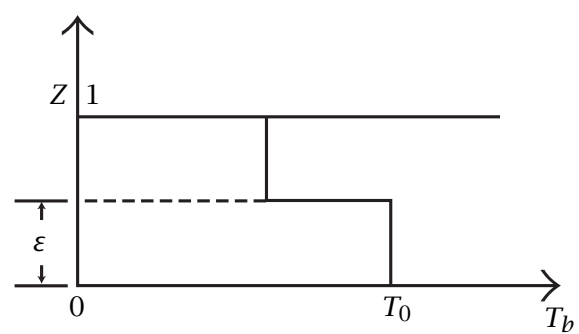

(d) Model-4: step function, $[f(z)=\delta(Z-\varepsilon)]$.

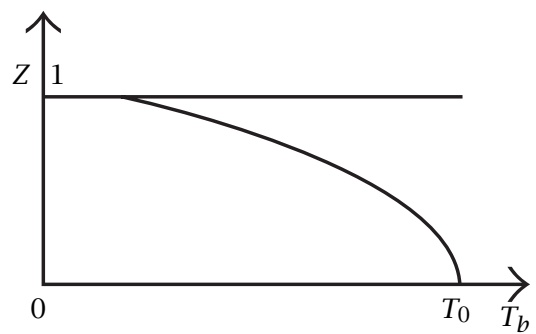

(f) Model-6: parabola, [ $f(z)=2 Z]$.

FIGURE 2.1. Basic state temperature profiles ( $\varepsilon=$ thermal depth) $(-d / \Delta T)$ $\left(d T_{b} / d z\right)=f(z)$.

We now nondimensionalize (3.1) using the following definitions:

$$
\begin{gathered}
\left(x^{*}, y^{*}, z^{*}\right)=\left(\frac{x}{d}, \frac{y}{d}, \frac{z}{d}\right), \quad\left(u^{*}, v^{*}, w^{*}\right)=\frac{\left(u^{\prime}, v^{\prime}, w^{\prime}\right)}{\left(\chi_{e} / d\right)}, \\
T^{*}=\frac{T^{\prime}}{\Delta T}, \quad p^{*}=\frac{p^{\prime}}{\left(\chi_{e} \mu_{f} / d^{2}\right)}, \quad t^{*}=\frac{t}{\left(d^{2} M / \chi_{e}\right)} .
\end{gathered}
$$


Substituting (3.2) in (3.1), we get

$$
\begin{gathered}
\left(1+\Gamma_{1} \frac{\partial}{\partial t}\right)\left(p_{, i}-R T \delta_{i 3}+\operatorname{Daq}_{i}\right)=\Lambda\left(1+\Gamma_{2} \frac{\partial}{\partial t}\right) q_{i, j j} . \\
\frac{\partial T}{\partial t}-w f(z)=T_{, j j},
\end{gathered}
$$

where

$$
\begin{aligned}
R & =\frac{\alpha g \Delta T d^{3}}{v \chi_{e}} & & \text { (Rayleigh number), } \\
\Gamma_{1} & =\frac{\lambda_{1}}{\left(M d^{2} / \chi_{e}\right)} & & \text { (Stress relaxation parameter) } \\
\Gamma_{2} & =\frac{\lambda_{2}}{\left(M d^{2} / \chi_{e}\right)} & & \text { (Strain retardation parameter) } \\
D a & =\frac{d^{2}}{K} & & \text { (Inverse Darcy number) } \\
\Lambda & =\frac{\mu^{\prime}}{\mu_{f}} & & \text { (Brinkman number) }
\end{aligned}
$$

and the asterisks have been dropped for simplicity. We now operate curl twice on (3.3) to get an equation involving only $w$. Such a procedure results in the following equation:

$$
\left(1+\Gamma_{1} \frac{\partial}{\partial t}\right)\left[-R \nabla_{1}^{2} T+D a \nabla^{2} w\right]=\Lambda\left(1+\Gamma_{2} \frac{\partial}{\partial t}\right) \nabla^{4} w,
$$

where $\nabla_{1}^{2}$ and $\nabla^{2}$ are the two- and three-dimensional Laplacian operators, respectively. Equations (3.4) and (3.5) are two equations in the two unknowns $T$ and $w$.

The infinitesimal perturbations $T$ and $w$ are assumed to be periodic waves and hence these permit normal mode solutions in the form (see Chandrasekhar [1])

$$
\left[\begin{array}{c}
T \\
w
\end{array}\right]=e^{\sigma t}\left[\begin{array}{c}
\theta(z) \\
W(z)
\end{array}\right] e^{i(k x+l y)},
$$

where the imaginary part of $\sigma$ is the frequency, $\theta(z)$ and $W(z)$ are the amplitudes, and $k$ and $l$ are the components of the wave number vector, that is, $\vec{a}=k i+l j$.

Substituting (3.6) into (3.4) and (3.5), we get

$$
\begin{aligned}
\sigma \theta-W f(z) & =\left(\frac{d^{2}}{d z^{2}}-a^{2}\right) \theta \\
R a^{2} \theta+D a\left(\frac{d^{2}}{d z^{2}}-a^{2}\right) W & =\Lambda \frac{\left(1+\Gamma_{2} \sigma\right)}{\left(1+\Gamma_{1} \sigma\right)}\left(\frac{d^{2}}{d z^{2}}-a^{2}\right)^{2} W
\end{aligned}
$$

where $\sigma$ is, in general, complex. Equations (3.7) and (3.8) are solved subject to the following boundary combinations:
(a) free-free isothermal,
(b) free-rigid isothermal,
(c) rigid-rigid isothermal. 
TABLE 3.1.

\begin{tabular}{|c|c|c|}
\hline Boundary combinations & Velocity $\left(W_{1}\right)$ & Temperature $\theta_{1}$ (isothermal) \\
\hline Free-free & $z-2 z^{3}+z^{4}$ & $z(1-z)$ \\
\hline Free-rigid & $2 z^{4}-5 z^{3}+3 z^{2}$ & $z(1-z)$ \\
\hline Rigid-rigid & $z^{2}-2 z^{3}+z^{4}$ & $z(1-z)$ \\
\hline
\end{tabular}

For free boundaries, we have

$$
W=\frac{d^{2} W}{d z^{2}}=0
$$

For rigid boundaries, we have

$$
W=\frac{d W}{d z}=0
$$

For isothermal condition, we have

$$
\theta=0
$$

Equations (3.7) and (3.8) are the Euler-Lagrange equations for the extremisation of the functional

$$
R=\frac{\left[D a\left\langle\left(D W_{1}\right)^{2}+a^{2} W_{1}\right\rangle+\frac{1+\sigma \Gamma_{2}}{1+\sigma \Gamma_{1}}\left\langle\left(D^{2} W_{1}\right)^{2}+2 a^{2}\left(D W_{1}\right)^{2}+a^{4} W_{1}^{2}\right\rangle\right]\left[\left\langle\left(D \theta_{1}\right)^{2}+\left(a^{2}+\sigma\right) \theta_{1}^{2}\right\rangle\right]}{a^{2}\left\langle W_{1} \theta_{1}\right\rangle\left\langle W_{1} \theta_{1} f(z)\right\rangle},
$$

where $W_{1}$ and $\theta_{1}$ are single-term Galerkin trial functions related to $W$ and $\theta$ by

$$
W=A W_{1}, \quad \theta=B \theta_{1},
$$

$A$ and $B$ being constants and $D=d / d z .\langle\cdots\rangle$ denotes the integration with respect to $z$ between $z=0$ and $z=1$.

It is now well established (see Nield [11]) that the trial functions (see Table 3.1) are appropriate for various boundary combinations.

Since the principle of exchange of stability is not valid in the present study, we can expect oscillatory instability and the stationary convection results are essentially the same as that of the Newtonian case

$$
R_{c}=\frac{1}{x_{5}}\left\{x_{1} x_{3} D a+\frac{x_{2} \Lambda}{\left(1+\omega^{2} \Gamma_{1}^{2}\right)}\left[x_{3}\left(1+\omega^{2} \Gamma_{1}^{2} Q\right)+x_{4} \Gamma_{1}(1-Q) \omega^{2}\right]\right\}+i \omega N,
$$

where

$$
\begin{aligned}
& N=\frac{1}{x_{5}}\left\{x_{1} x_{4} D a+\frac{x_{2} \Lambda}{\left(1+\omega^{2} \Gamma_{1}^{2}\right)}\left[x_{4}\left(1+\omega^{2} \Gamma_{1}^{2} Q\right)+x_{3} \Gamma_{1}(Q-1)\right]\right\}, \quad Q=\frac{\Gamma_{2}}{\Gamma_{1}}, \\
& x_{1}=\left\langle\left(D W_{1}\right)^{2}+a^{2} W_{1}^{2}\right\rangle, \quad x_{2}=\left\langle\left(D^{2} W_{1}\right)^{2}+2 a^{2}\left(D W_{1}\right)^{2}+a^{4} W_{1}^{2}\right\rangle, \\
& x_{3}=\left\langle\left(D \theta_{1}\right)^{2}+a^{2} \theta_{1}^{2}\right\rangle, \quad x_{4}=\left\langle\theta_{1}^{2}\right\rangle, \quad x_{5}=\left[\left\langle\left(W_{1} \theta_{1}\right)\right\rangle\left\langle W_{1} \theta_{1} f(z)\right\rangle\right] .
\end{aligned}
$$


Since $R_{c}$ is a real quantity, either $\omega=0$ (stationary) or $N=0(\omega \neq 0$, oscillatory). The latter condition yields

$$
\omega^{2}=-\left\{\frac{x_{1} x_{4} D a+x_{2} \Lambda\left[x_{4}+x_{3} \Gamma_{1}(Q-1)\right]}{x_{4} \Gamma_{1}^{2}\left[x_{1} D a+x_{2} \Lambda Q\right]}\right\} .
$$

Clearly $\omega^{2}$ is negative if $Q>1$ and in this case the principle of exchange of stability is valid. The results of Poulikakos [16] pertain to this case. This is, however, not of interest to us. We consider $Q<1$ in the paper and study oscillatory convection.

4. Results and discussions. In the paper, we study the effects of basic-state nonuniform temperature profile and viscoelasticity on the convective instability of a Boussinesquian fluid occupying a high-porosity porous medium. Six nonuniform basic temperature profiles are chosen for study and these have been presented in Figure 2.1. The grouping of nonuniform temperature profiles (presented in Table 4.2) can be made on the basis of their influence on instability in the case of the symmetric boundary combinations, namely, rigid-rigid and free-free.

TABLE 4.2 .

\begin{tabular}{|l|c|c|}
\hline \multicolumn{1}{|c|}{ Group 1 } & Group 2 & Group 3 \\
\cline { 1 - 2 } Linear $\left(R_{\mathcal{C}_{1}}\right)$ & $\begin{array}{r}\text { Piecewise linear }\left(R_{\mathcal{C}_{2}}\right) \\
\text { (heating from below) }\end{array}$ & \multirow{2}{*}{ Step function $\left(R_{\mathcal{C}_{4}}\right)$} \\
\cline { 1 - 2 } Inverted parabolic $\left(R_{\mathcal{C}_{5}}\right)$ & $\begin{array}{r}\text { Piecewise linear }\left(R_{\mathcal{C}_{3}}\right) \\
\text { (cooling from above) }\end{array}$ & \\
\hline Parabolic $\left(R_{\mathcal{C}_{6}}\right)$ & & \\
\hline
\end{tabular}

$\left(R_{c_{1}}\right)$ ( $i=1$ to 6$)$ in the above table are the critical Rayleigh numbers corresponding to the nonuniform basic temperature gradients described in Figure 2.1. We have $\left(R_{c_{1}}\right)=\left(R_{c_{5}}\right)=\left(R_{c_{6}}\right)$ and $\left(R_{\mathcal{C}_{2}}\right)=\left(R_{c_{3}}\right)$ for the symmetric boundary combinations. In the case of free-rigid boundaries (nonsymmetric boundary combination) no two $\left(R_{\mathcal{C}_{i}}\right)$ 's are the same. In this case, we find that

$$
\left(R_{\mathcal{C}_{4}}\right)<\left(R_{\mathcal{C}_{3}}\right)<\left(R_{\mathcal{C}_{2}}\right)<\left(R_{c_{1}}\right)<\left(R_{\mathcal{C}_{6}}\right)<\left(R_{\mathcal{C}_{5}}\right) .
$$

The reason for this is the symmetry/nonsymmetry of $W_{1} \theta_{1}$ about the mid-line of the channel. For all boundary combinations, step-function and inverted parabolic temperature profiles are, respectively, the most destabilizing and stabilizing distributions.

In the case of piecewise linear and step function profiles, the critical Rayleigh number $R_{c}$ depends on the thermal depth $\varepsilon$ and on the parameters of the problem. Table 4.3 provides information on the critical thermal depth that yields the critical eigenvalue for different boundary combinations with respect to all relevant basic temperature gradients. 
TABLE 4.3 .

\begin{tabular}{|c|c|c|c|c|c|c|}
\hline Boundary & \multicolumn{2}{|c|}{ Free-free } & \multicolumn{2}{|c|}{ Rigid-rigid } & \multicolumn{2}{|c|}{ Free-rigid } \\
\hline Profiles & $\varepsilon_{C}$ & Ratio of $R_{c_{i}}$ & $\varepsilon_{c}$ & Ratio of $R_{c_{i}}$ & $\varepsilon_{C}$ & Ratio of $R_{\mathcal{C}_{i}}$ \\
\hline Heating from below & 0.72 & $R_{c_{2}}=\frac{R_{c_{1}}}{1.1385}$ & 0.7064 & $R_{C_{2}}=\frac{R_{c_{1}}}{1.2487}$ & 0.7657 & $R_{C_{2}}=\frac{R_{c_{1}}}{1.1472}$ \\
\hline Cooling from above & 0.72 & $R_{c_{3}}=\frac{R_{c_{1}}}{1.1385}$ & 0.7064 & $R_{\mathcal{C}_{3}}=\frac{R_{\mathcal{C}_{1}}}{1.2847}$ & 0.6574 & $R_{c_{3}}=\frac{R_{c_{1}}}{1.2749}$ \\
\hline Step function & 0.50 & $R_{C_{4}}=\frac{R_{c_{1}}}{1.8214}$ & 0.5 & $R_{C_{4}}=\frac{R_{c_{1}}}{2.1875}$ & 0.5479 & $R_{\mathcal{C}_{4}}=\frac{R_{c_{1}}}{2.0289}$ \\
\hline Inverted parabolic & - & $R_{\mathcal{C}_{5}}=R_{\mathcal{C}_{1}}$ & - & $R_{C_{5}}=R_{C_{1}}$ & $\frac{1}{35}$ & $R_{C_{5}}=\frac{R_{c_{1}}}{0.9055}$ \\
\hline Parabolic & - & $R_{\mathcal{C}_{6}}=R_{\mathcal{C}_{1}}$ & - & $R_{\mathcal{C}_{6}}=R_{\mathcal{C}_{1}}$ & $\frac{1}{30}$ & $R_{\mathcal{C}_{6}}=\frac{R_{c_{1}}}{1.0565}$ \\
\hline
\end{tabular}

The information in Table 4.3 is in tune with what was reported by Currie [2]. We now discuss the viscoelastic effects on convection. From Figures $4.1 \mathrm{a}$ and $4.2 \mathrm{a}$, it is clear that the stress relaxation parameter $\Gamma_{1}$ and the strain retardation parameter $\Gamma_{2}$ have opposing influences. $\Gamma_{1}$ destabilizes whereas $\Gamma_{2}$ stabilizes. This result is true for all boundary combinations and for all the basic temperature profiles considered. Figures $4.1 \mathrm{~b}$ and $4.2 \mathrm{~b}$ indicate that the variation of cell size with $\Gamma_{1}$ at onset is not drastically different from its variations with $\Gamma_{2}$. It is the very significant variation of critical frequency with $\Gamma_{1}$ and $\Gamma_{2}$ that contributes to convection with very less comparative variations in cell size. This is clearly depicted in Figures 4.1c and 4.2c.

The effect of porous medium on convection shown in Figures 4.3 and 4.4 demonstrates the stabilizing nature of Darcy friction for all boundary combinations and all basic temperature profiles. However, the plot of $a_{c}^{2}$ versus $D a$ with respect to the rigid-rigid boundary combination needs special attention. The behaviour is opposite to that observed for free-rigid and free-free boundary combinations. This is due to the fact that rigid-rigid boundary combination is the most stable one compared with the other two and combined with the fact that the method used is a one-term Galerkin method with polynomial trial functions. The curve with respect to rigid-rigid is also steep (i.e., of maximum slope) compared with the other two, thus indicating that correction can possibly be sought by taking higher-order terms in the Galerkin method. Figure 4.5 delineates the stabilizing nature of the Brinkman number. This result was also observed in the Newtonian case. 


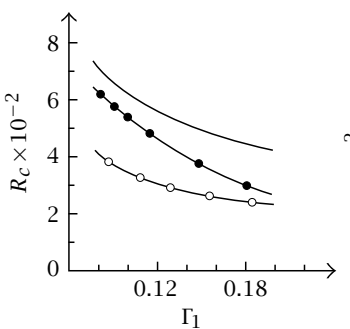

(a)

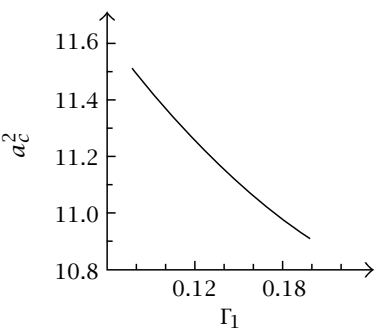

(b)

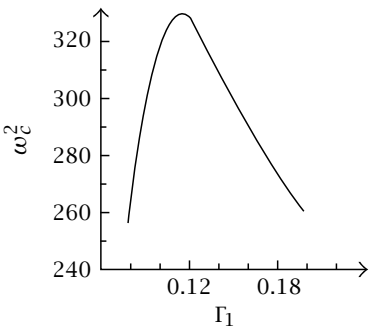

(c)

FIGURE 4.1. Plot of $R_{c}, a_{c}^{2}, \omega_{c}^{2}$ (respectively, (a), (b), and (c)) versus $\Gamma_{1}$ for different basic-state temperature profiles with respect to free-free velocity boundary combination. Parameter values: $Q=0.1, D a=5.0$, and $\Lambda=0.9$. $(-)$ linear; $(\bullet)$ heating from below; (॰) step function.

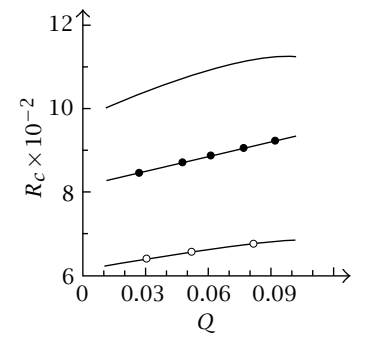

(a)

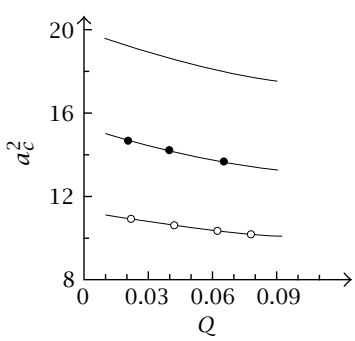

(b)

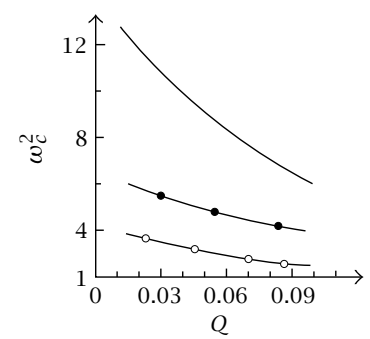

(c)

FIGURE 4.2. Plot of $R_{c}, a_{c}^{2}, \omega_{c}$ (respectively, (a), (b), and (c)) versus $Q$ for different velocity boundary combinations with respect to linear basic temperature profile, $f(z)=1$. Parameter values: $D a=5.0, \Lambda=0.9$, and $\Gamma_{1}=0.1$. (॰) free-free; $(\bullet)$ free-rigid; $(-)$ rigid-rigid.

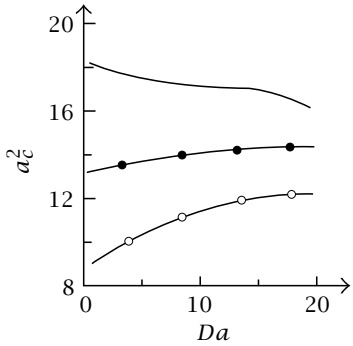

(a)

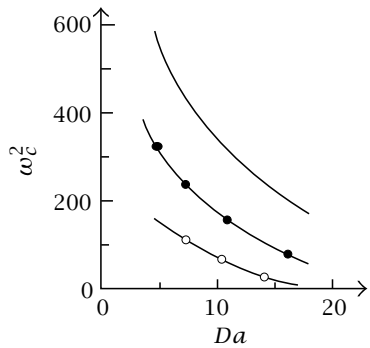

(b)

FIGURE 4.3. Plot of $D a$ versus (a) $a_{c}^{2}$ and (b) $\omega_{c}^{2}$ for different velocity boundary combinations with respect to linear basic temperature profile, $f(z)=1$. Parameter values: $\Lambda=0.9, Q=0.1$, and $\Gamma_{1}=0.1$. (॰) free-free; $(\bullet)$ free-rigid; (-) rigid-rigid. 


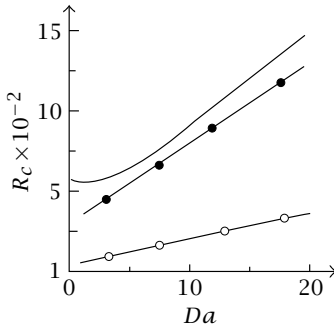

(a) Free-free.

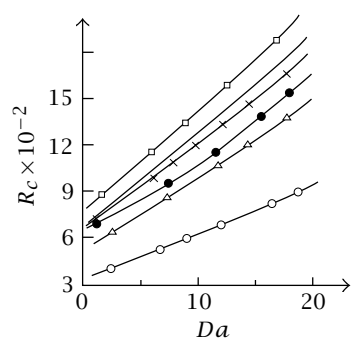

(b) Free-rigid.

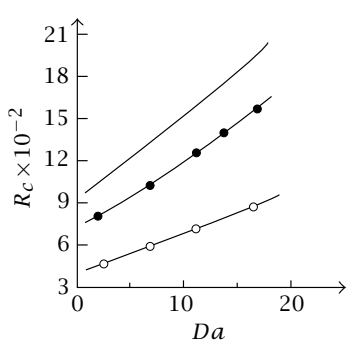

(c) Rigid-rigid.

FiguRE 4.4. Plot of $D a$ versus $R_{C}$ for different basic-state temperature profiles with respect to velocity boundary combination. Parameter values: $\Lambda=0.9, Q=0.1$, and $\Gamma_{1}=0.1$. (-) linear; (•) heating from below; (०) step function; $(\triangle)$ cooling from below; $(\square)$ inverted parabolic; $(\times)$ parabolic.

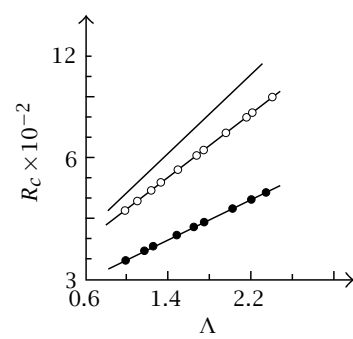

(a)

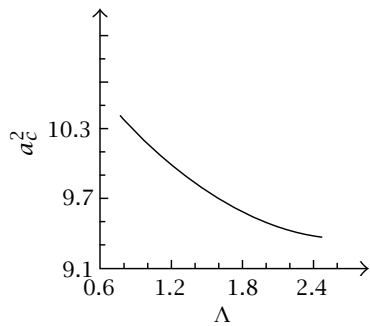

(b)

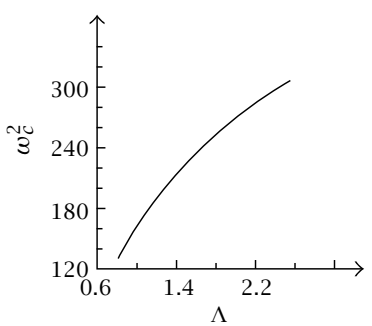

(c)

FiguRE 4.5. Plot of (a) $R_{c}$, (b) $a_{c}^{2}$, and (c) $\omega_{c}^{2}$ versus $\Lambda$ for different basicstate temperature profiles with respect to the free-free velocity boundary combination. Parameter values: $\Gamma_{1}=0.1, Q=0.1, D a=5.0$. (-) linear; (०) heating from below; $(\bullet)$ step function.

ACKNOWLEDGEMENT. The work was supported by the UGC-DSA and COSIST programmes at the Department of Mathematics, Bangalore University, India. The second author (C.V.S.) would like to acknowledge the support of the college administration for encouraging research work.

\section{REFERENCES}

[1] S. Chandrasekhar, Hydrodynamic and Hydromagnetic Stability, The International Series of Monographs on Physics, Clarendon Press, Oxford, 1961. MR 23\#B1270. Zbl 142.44103.

[2] I. G. Currie, The effect of heating rate on the stability of stationary fluids, J. Fluid Mech. 29 (1967), 337-347.

[3] I. A. Eltayeb, Nonlinear thermal convection in an elasticoviscous layer heated from below, Proc. Roy. Soc. London Ser. A 356 (1977), no. 1685, 161-176. MR 56\#17528. Zbl 359.76044.

[4] T. Green, Oscillating convection in an elastico-viscous liquid, Phys. Fluids 11(7) (1968), 1410-1413. 
[5] J. Harris, Rheology and Non-Newtonian Flow, vol. 338, Longman, New York, 1977. Zbl 445.76002.

[6] D. M. Herbert, On the stability of visco-elastic liquids in heated plane Couette flow, J. Fluid Mech. 17 (1963), 353-359. MR 29\#6762. Zbl 113.40501.

[7] G. Lauriat and V. Prasad, Natural convection is a vertical porous cavity: A numerical study for Brinkman-extended Darcy formulation, J. Heat Mass Transfer 109 (1987), 688695.

[8] T. S. Lundgren, Slow flow through stationary random beds and suspensions of spheres, J. Fluid Mech. 51 (1972), 273-299. Zbl 229.76067.

[9] J. Martinez-Mardones and C. Perez-Garcia, Linear instability in viscoelastic fluid convection, J. Phys. Condens. Matter 2 (1990), 1281-1283.

[10] D. A. Nield, Convection in a porous medium, Proc. CSIRO/DSIR Seminar on Convective Flows in Porous Media (New Zealand) (Wooding and White, eds.), DSIR, 1985.

[11] _ Throughflow effects in the Rayleigh-Benard convective instability problem, J. Fluid Mech. 185 (1987), 353-360.

[12] J. G. Oldroyd, On the formulation of rheological equations of state, Proc. Roy. Soc. London Ser. A. 200 (1950), 523-541. MR 11,703a.

[13] - The elastic and viscous properties of emulsions and suspensions, Proc. Roy. Soc. London Ser. A 128 (1953), 122-132. Zbl 050.41608.

[14] _ Non-Newtonian effects in steady motion of some idealized elastico-viscous liquids, Proc. Roy. Soc. London Ser. A 245 (1958), 278-297. MR 20\#605. Zbl 080.38805.

[15] H. M. Park and H. S. Lee, Non-linear hydrodynamical stability of viscoelastic fluid heated from below, J. Non-Newtonian Fluid Mech. 60 (1995), 1-34.

[16] D. Poulikakos, Double diffusive convection in a horizontal sparsely packed porous layer, Int. Comm. Heat Mass Transfer 13 (1986), 587-598.

[17] S. Rosenblat, Thermal convection in a viscoelastic liquid, J. Non-Newtonian Fluid Mech. 21 (1986), 201-223. Zbl 624.76007.

[18] N. Rudraiah, P. N. Kaloni, and P. V. Radhadevi, Oscillatory convection in a viscoelastic fluid through a porous layer heated from below, Rheol. Acta 28 (1989), no. 1, 4853. Zbl 676.76013.

[19] N. Rudraiah, P. V. Radhadevi, and P. N. Kaloni, Convection in a viscoelastic fluid-saturated sparsely packed porous layer, Can. J. Phys. 68(12) (1990), 1446-1453.

[20] N. Rudraiah and P. K. Srimani, Finite-amplitude cellular convection in a fluid-saturated porous layer, Proc. Roy. Soc. London Ser. A 373 (1980), no. 1753, 199-222. MR 82f:76037. Zbl 453.76043.

[21] M. Sokolov and R. I. Tanner, Convective stability of a general viscoelastic fluid heated from below, Phys. Fluids 15 (1972), 534-539. Zbl 247.76041.

[22] C. M. Vest and V. S. Arpaci, Overstability of a viscoelastic fluid layer heated from below, J. Fluid Mech. 36 (1969), 613-623. Zbl 175.24201.

Pradeep G. Siddheshwar: Department of Mathematics, Bangalore University, CenTRAL COLLEGE CAMPUS, BANGALORE-560 001, INDIA

E-mail address: pgsiddheshwar@hotmai 1 .com

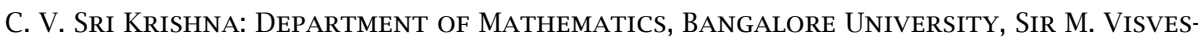
VARAYA, INSTITUTE OF TECHNOLOGY, BANGALORE-562 157, INDIA

E-mail address: cvsrikrishna@hotmai 1 .com 


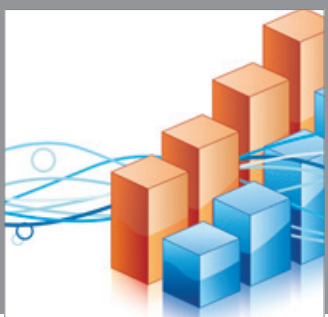

Advances in

Operations Research

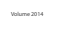

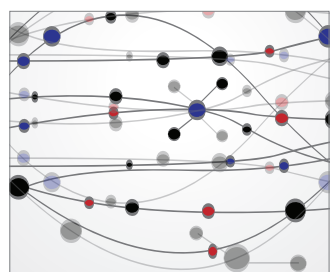

\section{The Scientific} World Journal
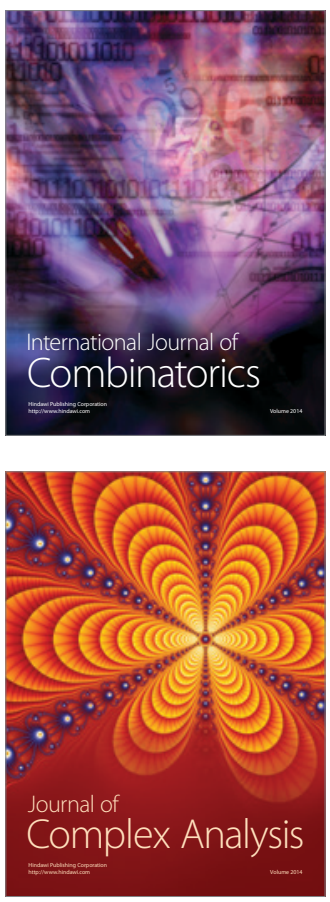

International Journal of

Mathematics and

Mathematical

Sciences
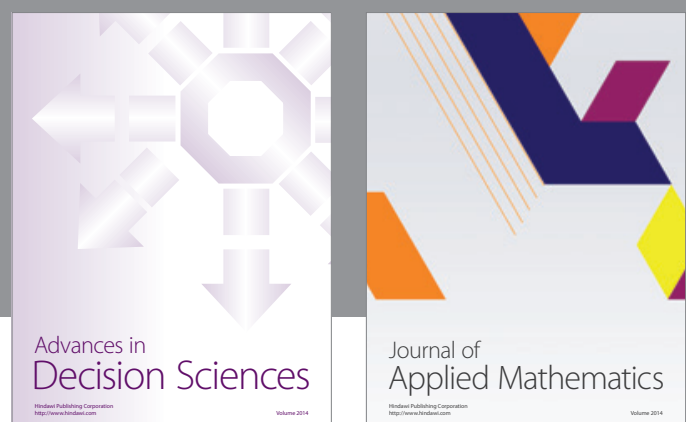

Journal of

Applied Mathematics
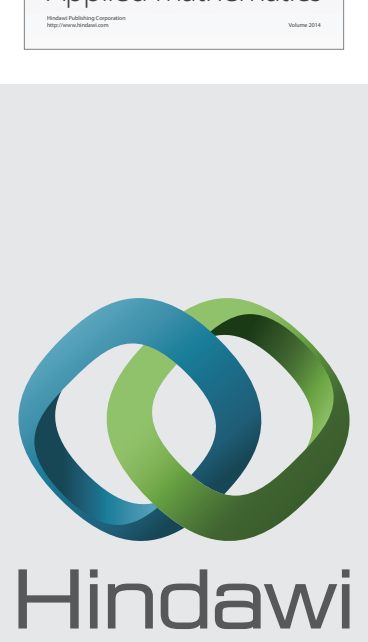

Submit your manuscripts at http://www.hindawi.com
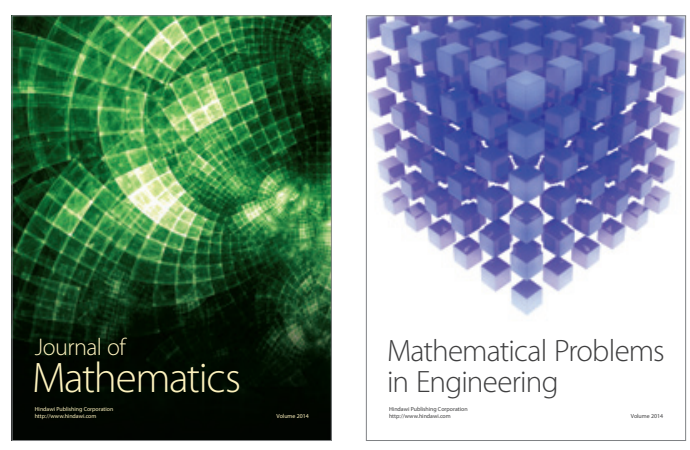

Mathematical Problems in Engineering
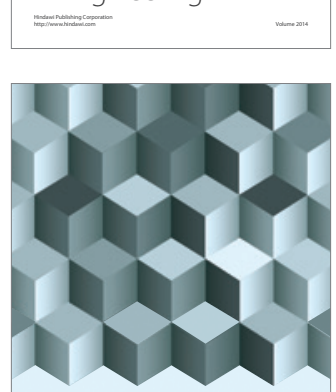

Journal of

Function Spaces
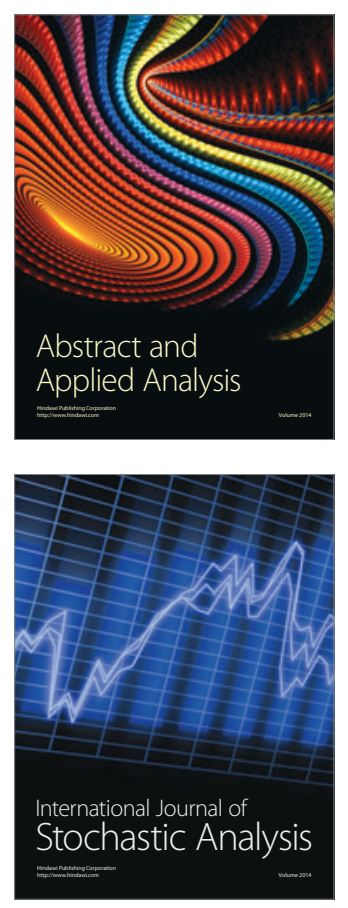

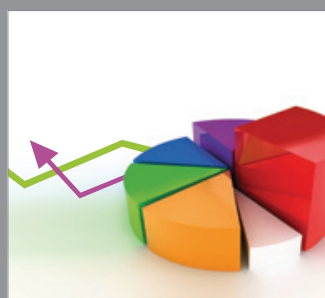

ournal of

Probability and Statistics

Promensencen
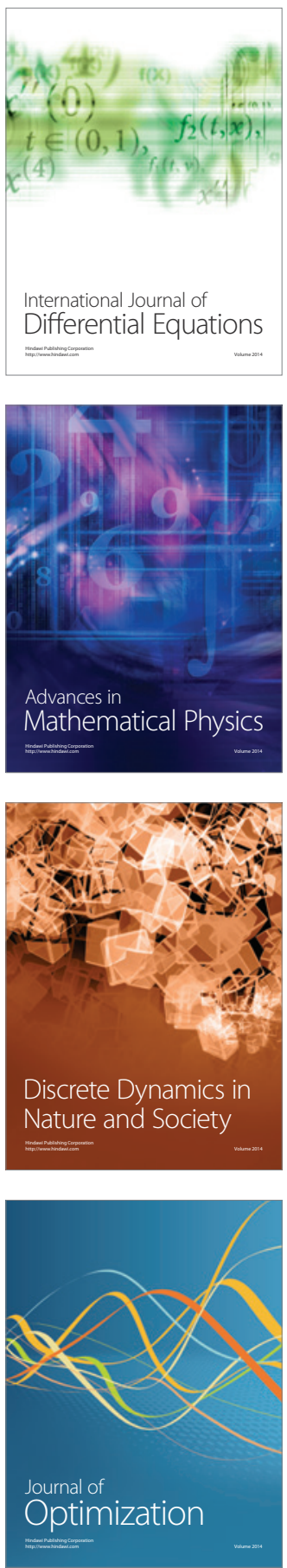